\title{
ON COMPLETE CONGRUENCE LATTICES OF COMPLETE MODULAR LATTICES
}

\author{
R. Freese, G. Grätzer, and E. T. Schmidt \\ University of Hawaii, University of Manitoba, \\ Mathematical Institute of the Hungarian Academy of Sciences
}

January 8, 1991

\begin{abstract}
The lattice of all complete congruence relations of a complete lattice is itself a complete lattice. In 1988, the second author announced the converse: every complete lattice $L$ can be represented as the lattice of complete congruence relations of some complete lattice $K$. In this paper we improve this result by showing that $K$ can be chosen to be a complete modular lattice.
\end{abstract}

1. Introduction. In this paper, we prove the following result:

Theorem. Every complete lattice $L$ is isomorphic to the lattice of complete congruence relations of a suitable complete modular lattice $K$.

For the history of earlier results, see the references G. Grätzer [3] and G. Grätzer and H. Lakser [4].

The basic idea is to replace the construction of G. Grätzer and H. Lakser [4] shown in Figure 1, with the construction illustrated by Figure 2. In both lattices, let $\Theta_{a}, \Theta_{b}$, and $\Theta_{c}$ denote the complete congruence relation generated by collapsing the covering pairs marked by $a, b$, and $c$, respectively. Then $\Theta_{a} \vee \Theta_{b}=\Theta_{c}$ holds in the lattice of complete congruence relations - note that it does not hold in the lattice of congruence relations. While both lattices are complete, only the lattice of Figure 2 is modular.

2. Notation. $L$ is the complete lattice we want to represent in the Theorem. We denote by 0 and 1 the zero and unit element of $L$, respectively. If $|L|=1$, it is trivial to represent $L$. We shall, henceforth, assume that $|L| \geq 2$.

1991 Mathematics Subject Classification. Primary 06B10; Secondary 06C05.

Key words and phrases. Complete lattice, modular lattice, complete congruence, congruence lattice.

The research of the first author was supported by NSF grant no. DMS89-01756.

The research of the second author was supported by the NSERC of Canada.

The research of the third author was supported by the Hungarian National Foundation for Scientific Research, under Grant No. 1813. 


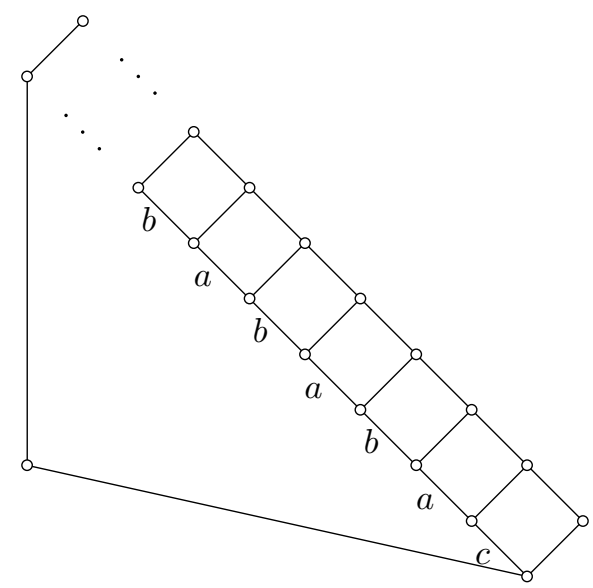

Figure 1

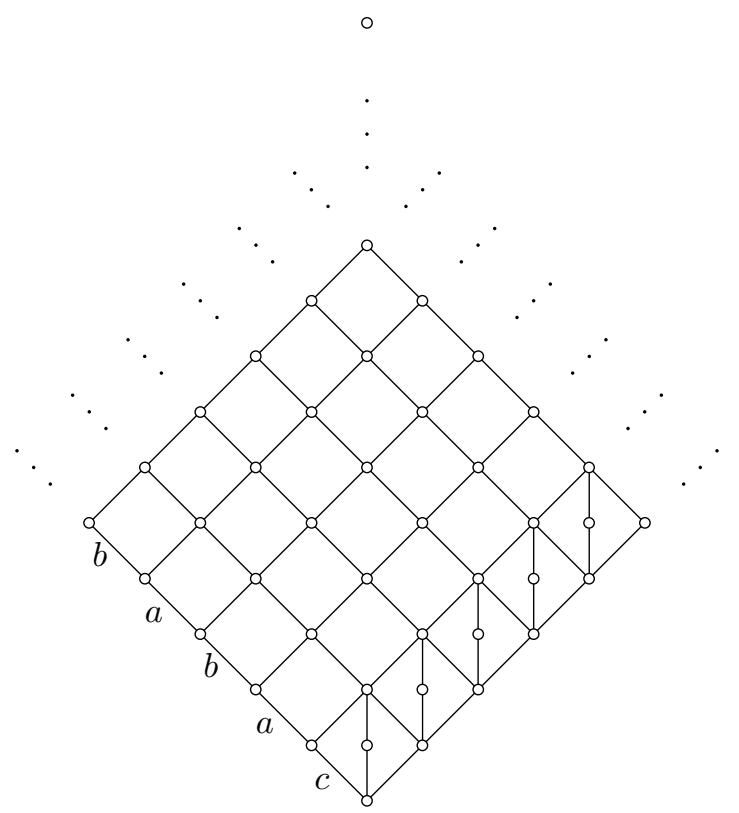

FiguRE 2

We shall be dealing with nonempty subsets $X \subseteq L-\{0\}$. Let us write

$$
X=\left\{x_{\gamma} \mid \gamma<\zeta^{X}\right\},
$$


where $1 \leq \zeta^{X} \leq \zeta=|L-\{0\}|$ (cardinals are regarded as initial ordinals). Let $\left\{X^{\delta} \mid \delta<\chi\right\}$ denote the family of all such sets; the elements of $X^{\delta}$ are well-ordered:

$$
X^{\delta}=\left\{x_{\gamma}^{\delta} \mid \gamma<\zeta^{\delta}\right\}
$$

Since $|L| \geq 2$, it follows that there is at least one such $X$, that is, $0<\chi$.

$\mathfrak{M}_{3}$ denotes the five-element modular nondistributive lattice; $\mathbf{1}$ and $\mathbf{2}$ denote the one-element and two-element chains, respectively.

Let $\alpha$ be an ordinal, and for $\gamma<\alpha$, let $A_{\gamma}$ be a lattice. We denote by

$$
\Sigma\left(A_{\gamma} \mid \gamma<\alpha\right)
$$

the ordinal sum of the $A_{\gamma}$ for $\gamma<\alpha$; for two components, $A$ and $B, A+B$ denotes the ordinal sum of $A$ and $B$ (we place $B$ on top of $A$ ).

For ordinals $\alpha, \beta$, the ordinal product $\alpha \times \beta$ is the set

$$
\{\langle\gamma, \delta\rangle \mid \gamma<\alpha, \delta<\beta\}
$$

ordered lexicographically, i.e., $\left\langle\gamma_{1}, \delta_{1}\right\rangle \leq\left\langle\gamma_{2}, \delta_{2}\right\rangle$ if and only if $\gamma_{1}<\gamma_{2}$ or $\gamma_{1}=\gamma_{2}$ and $\delta_{1} \leq \delta_{2}$.

For a lattice $A$, let Ip $A$ denote the the set of prime intervals in $A$, that is, the set of all intervals $\mathfrak{p}=[u, v]$, where $u \prec v$. If $x$ is an element of a well-ordered chain, $x<1$, then $x^{*}$ is the cover of $x$.

For a (prime) interval $\mathfrak{p}=[u, v]$ in the lattice $A$, we shall denote by $\Theta_{A}(\mathfrak{p})$ the congruence relation generated by the prime interval $\mathfrak{p}$. If $A$ is understood, we use the notation $\Theta(\mathfrak{p})$. In case $A$ is complete, $\Theta_{A}^{c}(\mathfrak{p})$ (or, simply, $\Theta^{c}(\mathfrak{p})$ ) will denote the complete congruence relation generated by $\mathfrak{p}=[u, v]$.

For a complete lattice $A$, the complete congruence lattice of $A$ is denoted by $\operatorname{Con}^{c} A$; the lattice operations in $\operatorname{Con}^{c} A$ are denoted by $\wedge, \vee^{c}$, and the infinite variants by $\Lambda$ and $\bigvee^{c}$. Note that $\Lambda$ and $\Lambda$ are set intersection.

Following S.-K. Teo [8], a coloring of a chain $C$ is a map

$$
\varphi: \operatorname{Ip} C \rightarrow L-\{0\}
$$

where $L$ is a complete lattice. If $\mathfrak{p} \in \operatorname{Ip} C$ and $\mathfrak{p} \varphi=a$, one should think of $\Theta_{K}^{c}(\mathfrak{p})$ as the complete congruence representing $a \in L-\{0\}$ in some extension $K$ of $C$.

If $\varphi$ is a coloring of a well-ordered chain $C$, it will be convenient to define the color of an element $x \in C, x<1$ - denoted by $x \varphi$-as $\left[x, x^{*}\right] \varphi$.

For a complete lattice $\mathrm{A}$ and a complete congruence $\Theta$ on $A$, we define the prime interior of $\Theta$, denoted by pi $\Theta$, as follows:

$$
\text { pi } \Theta=\bigvee^{c}\left(\Theta^{c}(\mathfrak{p}) \mid \mathfrak{p} \in \operatorname{Ip} A, \Theta(\mathfrak{p}) \leq \Theta\right) .
$$

In words, the prime interior of a complete congruence $\Theta$ is the smallest complete congruence collapsing all prime intervals collapsed by $\Theta$.

Let $A$ be a complete lattice which is strongly atomic, that is, for any $w, z \in A$, $w<z$, there is an element $p \in A$ satisfying $w \prec p \leq z$. In G. Grätzer and H. Lakser [4], the following observation was made: 
Lemma 1. In a strongly atomic complete lattice A, the equality

$$
\text { pi } \Theta=\Theta
$$

holds for any complete congruence $\Theta$ of A.

We refer the reader to Crawley and Dilworth [1] and G. Grätzer [2] for the standard notation in lattice theory.

The next lemma gives a simple characterization of complete congruences on a complete lattice. Recall that a lattice homomorphism from $K$ to $K^{\prime}$ is said to be a bounded homomorphism if the set of inverse images of each element of $K^{\prime}$ has a least and greatest element, see R. McKenzie [7].

Lemma 2. Let $\Theta$ be a congruence on a complete lattice $K$. Then the following are equivalent.

(1) $\Theta \in \operatorname{Con}^{c}(K)$, i.e., $\Theta$ is a complete congruence.

(2) Each block of $\Theta$ is a complete sublattice of $K$.

(3) Each block of $\Theta$ is a complete lattice.

(4) Each block of $\Theta$ is bounded, i.e., has a least and greatest element.

(5) The natural map $K \rightarrow K / \Theta$ is a bounded homomorphism.

Proof. Since $K$ is a complete lattice and blocks of any congruence are always convex sublattices, it is easy to see that (2)-(5) are equivalent. It is also easy to see that (1) implies (2).

Now assume (2) and suppose that $x_{i} \Theta y_{i}$ for $i \in I$. Let $x=\bigvee x_{i}$ and $y=\bigvee y_{i}$. Then $x=x \vee x_{i} \Theta x \vee y_{i}$. Since we are assuming $x / \Theta$ is complete, this implies $x \Theta x \vee y$. Similarly $y \Theta x \vee y$.

3. The chains $X^{\dagger}$ and $C$. The lattice $K$ of the Theorem is defined using three chains, $C, D$, and $E$. In this section, we define the chains $X^{\dagger}$, and build $C$ up from the chains $X^{\dagger}$.

Let $X=\left\{x_{\gamma} \mid \gamma<\zeta^{X}\right\} \subseteq L-\{0\}$ be given.

We construct the chain $X^{\dagger}$ - see Figure 3 - as follows:

$$
\mathbf{1}+(\omega \times X)+\mathbf{1} .
$$

The zero of $X^{\dagger}$ is $0^{X}$ and the unit element is $1^{X}$; the other elements of $X^{\dagger}$ are

$$
j^{X}=\left\langle 0, x_{0}\right\rangle<\cdots<\left\langle 0, x_{\gamma}\right\rangle<\cdots<\left\langle i, x_{0}\right\rangle<\cdots<\left\langle i, x_{\gamma}\right\rangle<\ldots,
$$

for $\gamma<\zeta^{X}$ and $i<\omega$. For $\left(X^{\delta}\right)^{\dagger}$, we use the notation $0^{\delta}, j^{\delta}$, and $1^{\delta}$.

We define a coloring $\varphi^{X}$ (for $\left(X^{\delta}\right)^{\dagger}$, denoted by $\varphi^{\delta}$ ) on $X^{\dagger}$ as illustrated by Figure 3:

$$
\begin{aligned}
{\left[0^{X}, j^{X}\right] \varphi^{X} } & =\bigvee X ; \\
{\left[\left\langle i, x_{\gamma}\right\rangle, u\right] \varphi^{X} } & =x_{\gamma}, \quad \text { for } i<\omega \text { and } \gamma<\zeta^{X},
\end{aligned}
$$

where

$$
u= \begin{cases}\left\langle i, x_{\gamma+1}\right\rangle, & \text { if } \gamma+1<\zeta^{X} \\ \left\langle i+1, x_{0}\right\rangle, & \text { if } \gamma+1=\zeta^{X}\end{cases}
$$


$1^{X}$ 。

$$
\left.\begin{array}{l}
\left\langle 1, x_{2}\right\rangle \\
\left\langle 1, x_{1}\right\rangle \\
\left\langle 1, x_{0}\right\rangle
\end{array}\right\} \begin{aligned}
& x_{1} \\
& x_{0}
\end{aligned}
$$

$X^{\dagger}$

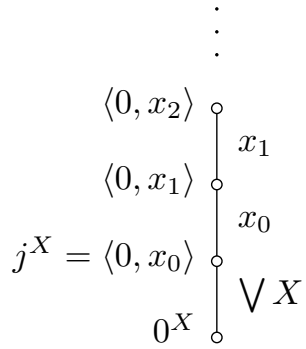

$1^{\delta} \circ$

$\left(X^{\delta}\right)^{\dagger}$

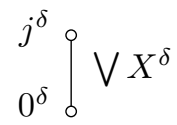

\section{FIGURE 3}

The chain $X^{\dagger}$ is well-ordered, and $1^{X}$ is a limit ordinal. The following observation is crucial: in $X^{\dagger}$, for every $j^{X} \leq u<1^{X}$ and for every $\gamma<\zeta^{X}$, there is a prime interval $\mathfrak{p}$ in $\left[u, 1^{X}\right]$ such that $\mathfrak{p} \varphi=x_{\gamma}$.

Next, we define the well-ordered chain $C$ and the coloring $\varphi_{C}$.

For every $X^{\delta}, \delta<\chi$, we construct the chain $\left(X^{\delta}\right)^{\dagger}$, and we form the ordinal sum:

$$
C= \begin{cases}\mathbf{1}+\Sigma\left\{\left(X^{\delta}\right)^{\dagger} \mid \delta<\chi\right\}+\mathbf{1}, & \text { if } \chi \text { is limit; } \\ \mathbf{1}+\Sigma\left\{\left(X^{\delta}\right)^{\dagger} \mid \delta<\chi\right\}, & \text { if } \chi \text { is not limit; }\end{cases}
$$

see Figure 4 . The zero and unit element of $C$ is denoted by $0^{C}$ and $1^{C}$, respectively. Observe that if $\chi$ is not limit, that is, $\chi=\chi_{0}+1$, then $1^{C}=1^{\chi_{0}}$.

$C$ is a well-ordered chain. We define a coloring $\varphi_{C}$ of $C$. For a prime interval $\mathfrak{p}$ of $C$, let

$$
\mathfrak{p} \varphi= \begin{cases}\mathfrak{p} \varphi^{\delta}, & \text { if } \mathfrak{p} \in \operatorname{Ip}\left(X^{\delta}\right)^{\dagger}, \text { for some } \delta<\chi \\ 1, & \text { if } \mathfrak{p}=\left[0^{C}, 0^{0}\right] ; \\ 1, & \text { if } \mathfrak{p}=\left[1^{\delta}, 0^{\delta+1}\right], \text { for some } \delta<\chi\end{cases}
$$

4. Two more chains. In this section, we define the colored chains $D$ and $E$.

Let the chain $D$ be $(\chi \times \omega)+\mathbf{1}$, with zero $0^{D}$ and unit $1^{D}$ - see Figure 5 . We color $D$ as follows:

Let $d \in D$; then $d=\langle\delta, n\rangle$, where $\delta<\chi$ and $n<\omega$. Define $\left[d, d^{*}\right] \varphi_{D}=d \varphi_{D}=$ $\bigvee X^{\delta}$, where $\bigvee X^{\delta}$ is formed in the complete lattice $L$. We shall use the notation 


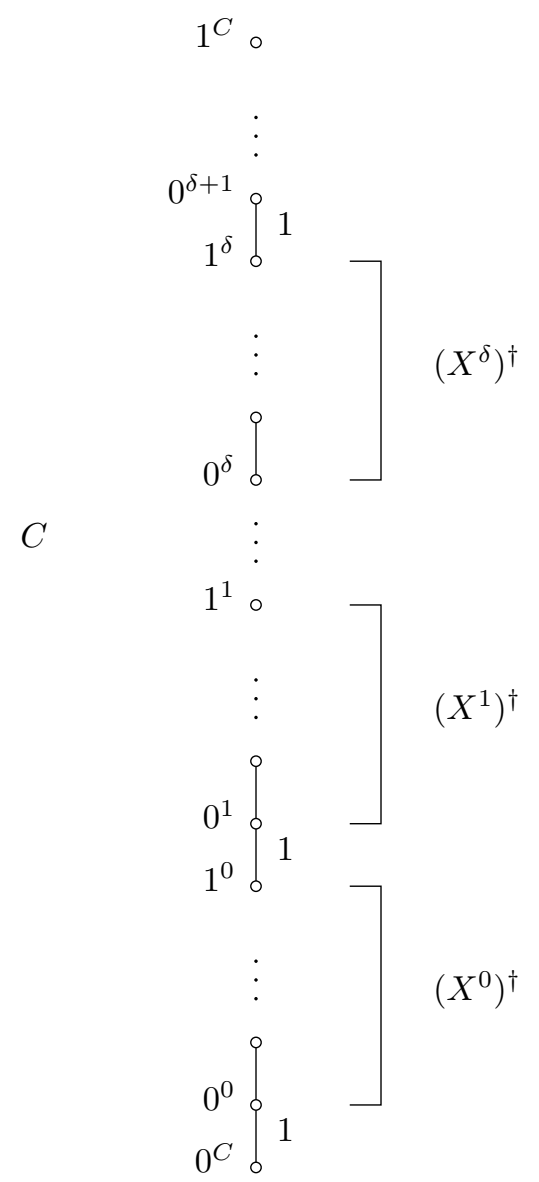

FIGURE 4

$D^{\delta}$ to denote the subchain of elements: $\langle\delta, 0\rangle,\langle\delta, 1\rangle, \ldots,\langle\delta, n\rangle, \ldots$, and $\langle\delta+1,0\rangle$; the zero and unit of $D^{\delta}$ will be denoted by $0^{D^{\delta}}$ and $1^{D^{\delta}}$, respectively. Note that $0^{D^{\delta}}=\langle\delta, 0\rangle$ and $1^{D^{\delta}}=\langle\delta+1,0\rangle$.

The chain $E$ is $\zeta+\mathbf{1}$, where $L-\{0\}=\left\{x_{\gamma} \mid \gamma<\zeta\right\}$ with zero $0^{E}$ and unit $1^{E}$. We color $E$ as follows: $\left[x_{\gamma}, x_{\gamma}^{*}\right] \varphi_{E}=x_{\gamma} \varphi_{E}=x_{\gamma}$, for $\gamma<\zeta$.

5. The augmented grid $\hat{K}$. It is convenient to construct a lattice $\hat{K}$ first; the lattice $K$ will be a sublattice of $K$.

The direct product $C \times D \times E$ we shall call the "grid;" its elements are the "grid elements." The prime intervals of $C \times D \times E$ are colored in the obvious way. For example, if $\mathfrak{p}$ is a prime interval of $C$ of color $a$, then the prime interval $\mathfrak{p} \times\{d\} \times\{e\}$ of $C \times D \times E$ has color $a$.

We use the notation $o=\left\langle 0^{C}, 0^{D}, 0^{E}\right\rangle$ and $i=\left\langle 1^{C}, 1^{D}, 1^{E}\right\rangle$. 


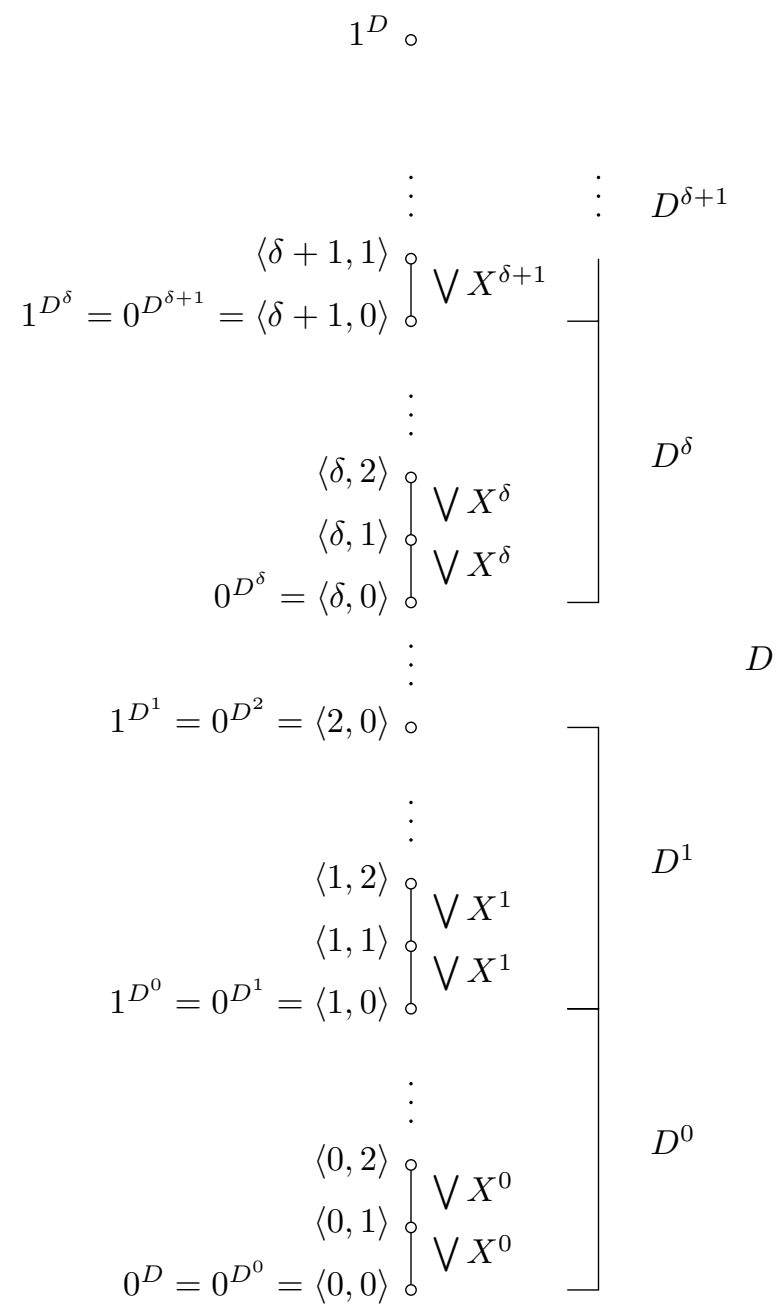

Figure 5

To construct $\hat{K}$, we augment the grid with two types of elements. Let $p=\langle c, d, e\rangle$ be a grid element; if $c \varphi_{C}=d \varphi_{D}$, then the elements

$$
\langle c, d, e\rangle,\left\langle c^{*}, d, e\right\rangle,\left\langle c, d^{*}, e\right\rangle,\left\langle c^{*}, d^{*}, e\right\rangle
$$

form a "monochromatic prime square" with respect to the axis $E$ over $p$. Monochromatic squares with respect to the axes $C$ and $D$ are defined similarly.

If there is a monochromatic prime square with respect to the axis $E$ over $p$, then we add an element $m(p, E)$ as shown in Figure 6. The elements

$$
\langle c, d, e\rangle,\left\langle c^{*}, d, e\right\rangle,\left\langle c, d^{*}, e\right\rangle, m(p, E),\left\langle c^{*}, d^{*}, e\right\rangle
$$




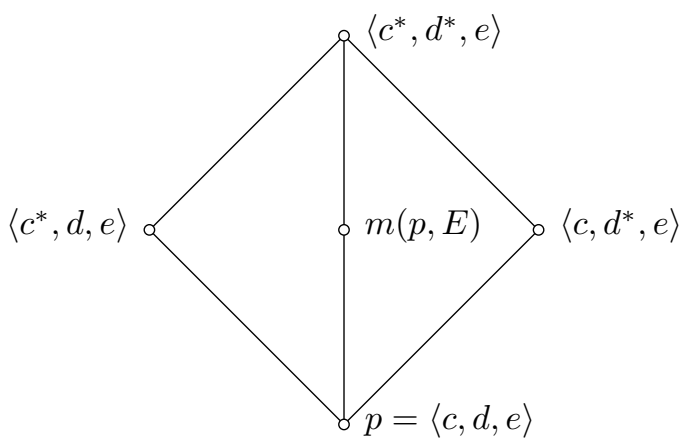

Figure 6

form a sublattice in the augmented lattice isomorphic to $\mathfrak{M}_{3}$. If $p^{\prime}=\left\langle c, d, e^{\prime}\right\rangle$ and $e<e^{\prime}$, then $m(p, E)<m\left(p^{\prime}, E\right)$. We proceed similarly for $C$ and $D$.

Given a grid element $p=\langle c, d, e\rangle$ satisfying $c \varphi_{C}=d \varphi_{D}=e \varphi_{E}$, then the interval

$$
\left[\langle c, d, e\rangle,\left\langle c^{*}, d^{*}, e^{*}\right\rangle\right]
$$

of the grid is called a "monochromatic prime cube" over $p$. This interval is isomorphic to $\mathbf{2}^{3}$. We use the notation

$$
p_{C}=\left\langle c^{*}, d, e\right\rangle, \quad p_{D}=\left\langle c, d^{*}, e\right\rangle, \quad p_{E}=\left\langle c, d, e^{*}\right\rangle .
$$

In a monochromatic prime cube over $p$ we have already placed six new elements:

$$
m(p, C), m(p, D), m(p, E), m\left(p_{C}, C\right), m\left(p_{D}, D\right), m\left(p_{E}, E\right) .
$$

Finally, we add two more elements to this interval: $a(p)$ and $b(p)$ as shown in Figure 7 , so that the eight elements of the prime cube, the six elements we have added before, and the two new elements form a lattice isomorphic to the subspace lattice of a Fano plane, i.e., the lattice of subspaces of a 3 dimensional vector space over $\operatorname{GF}(2)$.

We denote by $\hat{K}$ the grid $C \times D \times E$ augmented by all these elements.

Lemma 3. $\hat{K}$ is a complete modular lattice.

There are three easy ways to prove this. Firstly, $\hat{K}$ can be embedded in the subspace lattice of a vector space of high enough dimension over GF(2); since the subspace lattice is complete and modular and the embedding is complete, the lemma follows. Secondly, we can start from $\mathbf{2}^{3}, \mathfrak{M}_{3} \times \mathbf{2}$, and the subspace lattice of the Fano plane, and build up $\hat{K}$ by Dilworth gluing, the formation of ideal lattices, and direct limits; the lemma again follows. Thirdly, one can describe directly the joinand meet-tables of $\hat{K}$. We leave the details to the reader. 


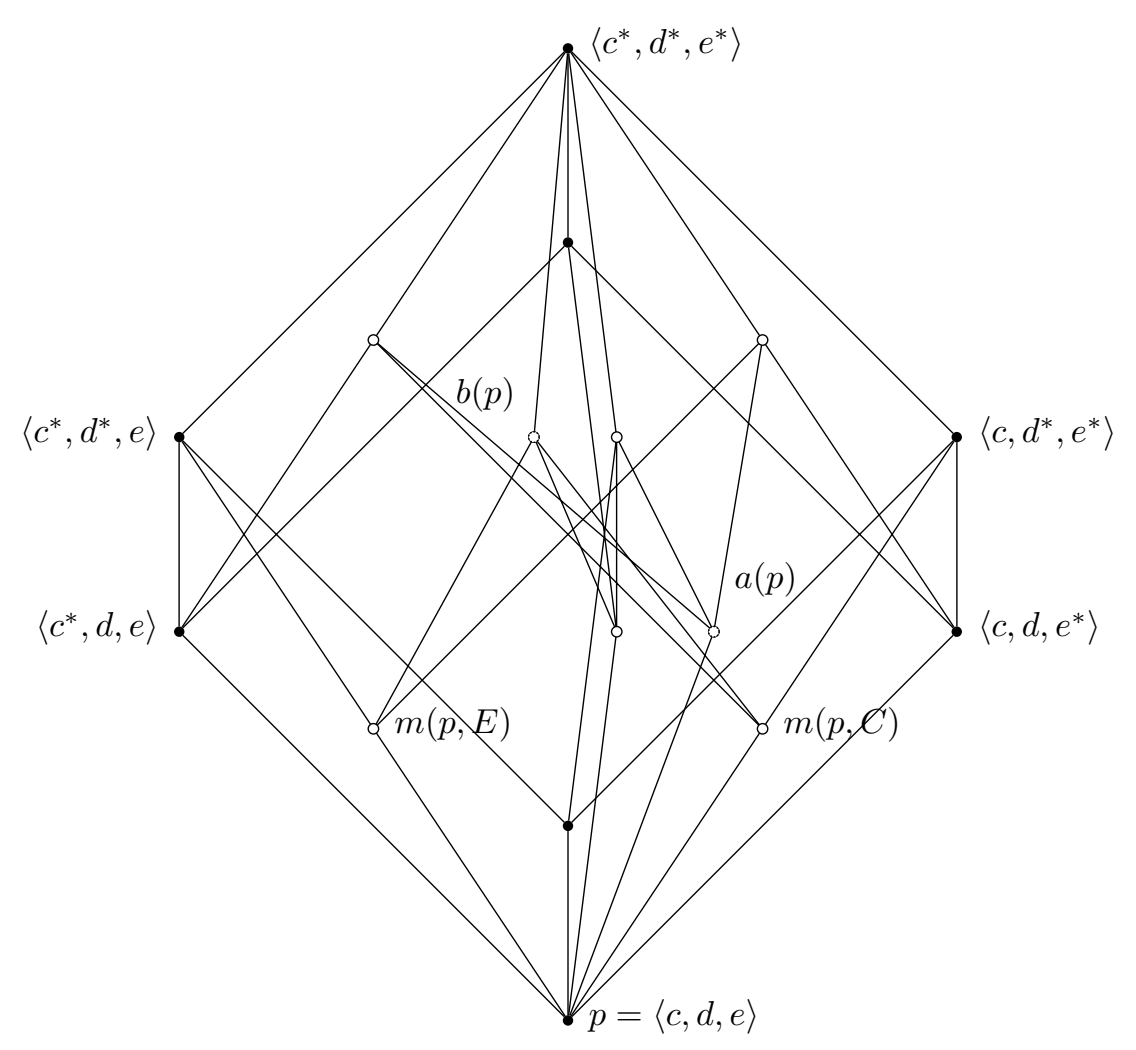

Figure 7

6. The lattice $K$. Now we are ready to define the lattice $K$. We define it piecewise.

Let $\delta<\chi$. We define the lattice $\mathfrak{M}_{\delta}$ (see Figure 8 for a schematic representation) as the convex sublattice of $\hat{K}$ generated by

$$
\begin{gathered}
\left(\left(X^{\delta}\right)^{\dagger} \times D^{\delta} \times E-\left\{\langle x, y, z\rangle \mid x=1^{\delta} \text { or } y=1^{D^{\delta}}\right\}\right) \\
\cup\left\{\left\langle 1^{\delta}, 1^{D^{\delta}}, z\right\rangle \mid z \in E\right\} .
\end{gathered}
$$

Observe that

$$
\left(\left(X^{\delta}\right)^{\dagger} \times D^{\delta}-\left\{\langle x, y\rangle \mid x=1^{\delta} \text { or } y=1^{D^{\delta}}\right\}\right) \cup\left\{\left\langle 1^{\delta}, 1^{D^{\delta}}\right\rangle\right\}
$$

is the lattice of Figure 2 in the special case $X^{\delta}=\{a, b\}$ and $c=a \vee b$.

The above set is a sublattice of the grid, and $\mathfrak{M}_{\delta}$ consists of these elements and the additional elements we defined in $\S 5$ for these elements. Finally, we define the lattice $K$ in the Theorem as a sublattice of $\hat{K}$ :

$$
K=\bigcup\left(\mathfrak{M}_{\delta} \mid \delta<\chi\right) \cup\left\{\left\langle 1^{C}, 1^{D}, x\right\rangle \mid x \in E\right\},
$$




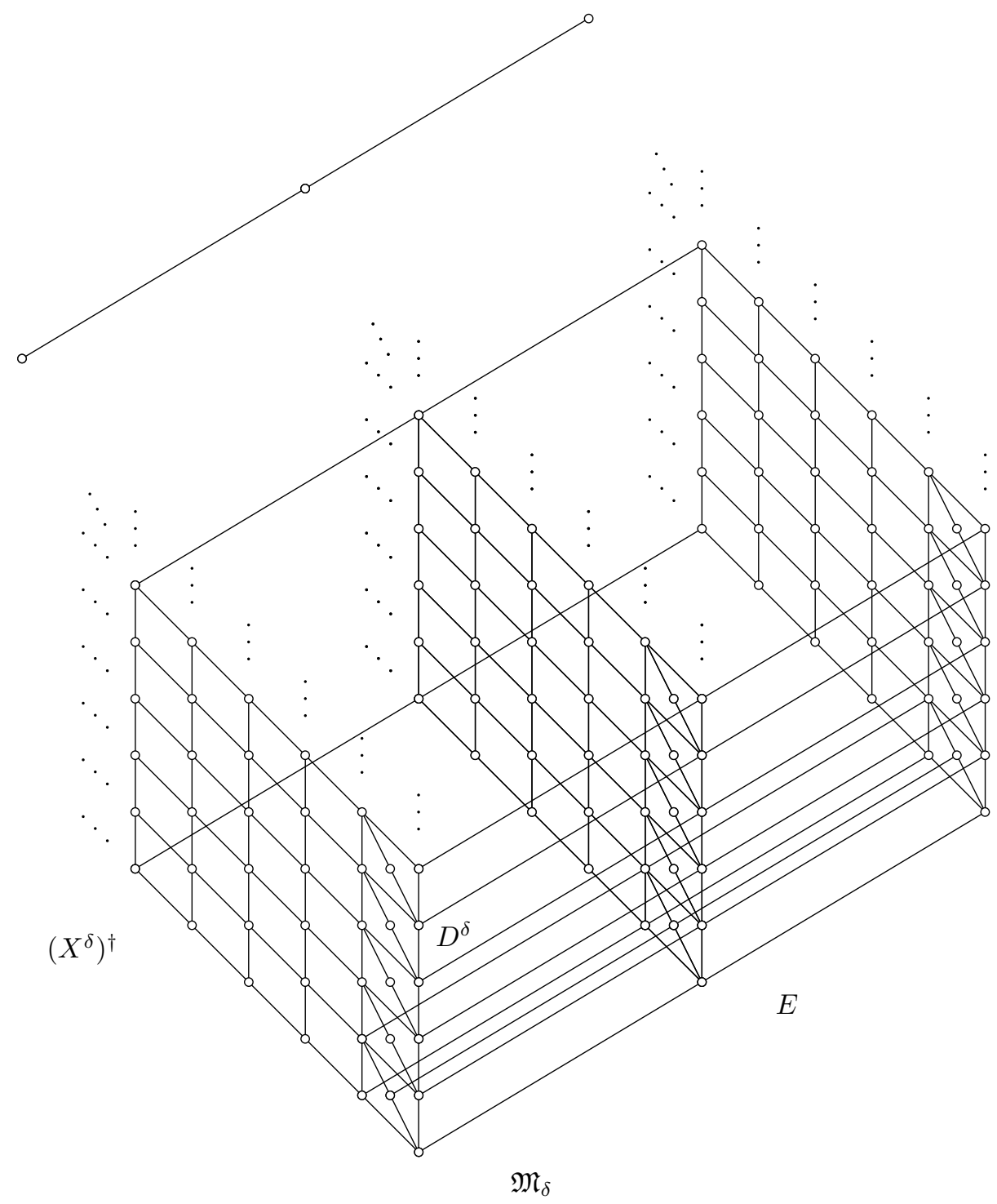

FiguRE 8

see Figure 9:

Lemma 4. $K$ is a complete modular lattice.

$K$ is a sublattice of $\hat{K}$, hence it is a modular lattice. $K$ is a closure system in $\hat{K}$, hence it is a complete lattice. Observe that $K$ is not a complete sublattice of $\hat{K}$. Indeed, let $a$ be the complete join of a subset $S$ of $K$ in $\hat{K}$. If $a \in K$, then it is the complete join of $S$ in $K$. Otherwise, $a$ must be of the form $\langle x, y, z\rangle$, where 


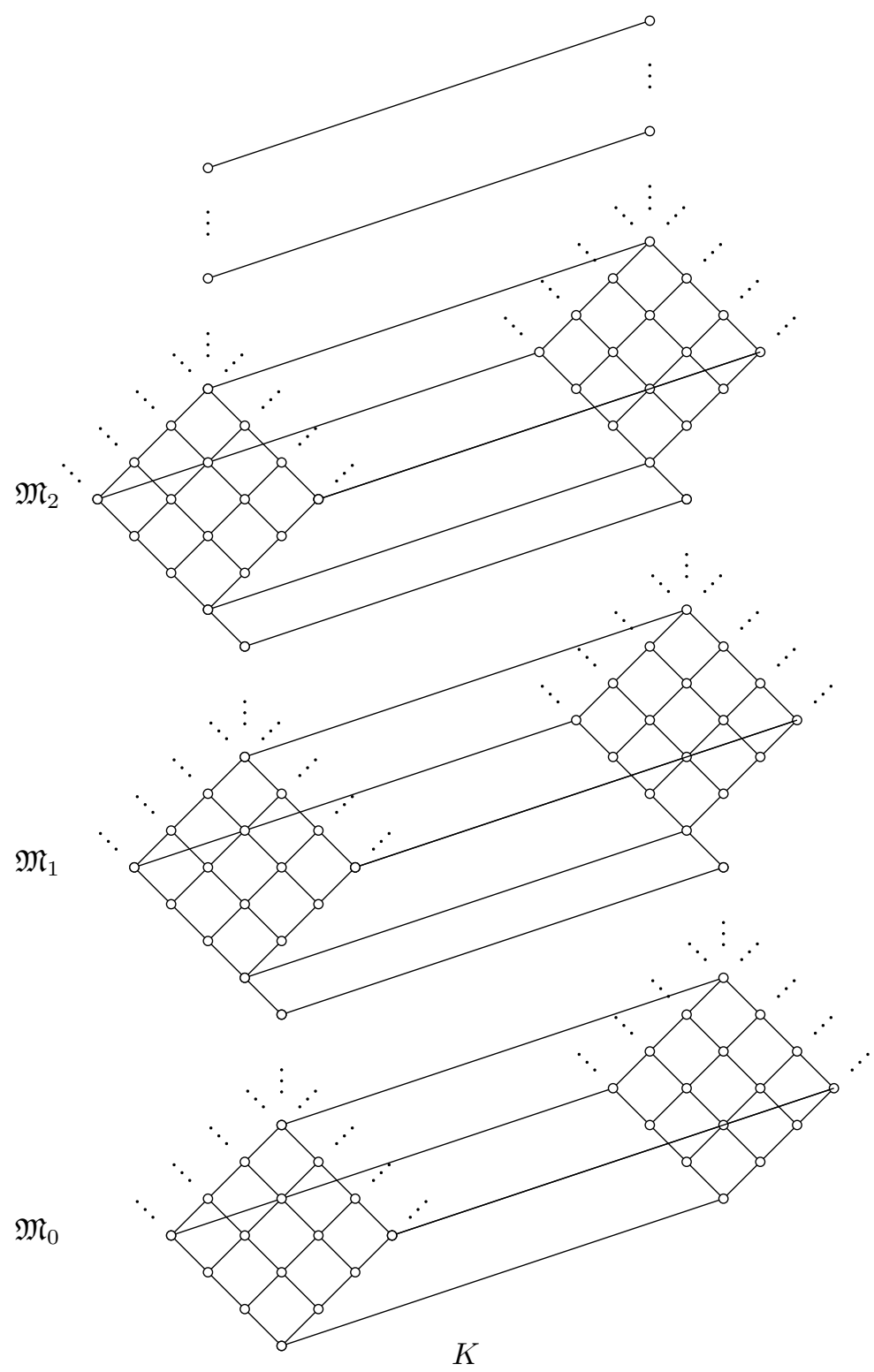

FiguRE 9

$x=1^{\delta}$ or $y=1^{D^{\delta}}$. Then $\bigvee S=\left\langle 1^{\delta}, 1^{D^{\delta}}, z\right\rangle$ in $K$.

7. The congruence $\Theta_{a}$. As the final step in preparing for the proof of the Theorem, for every $a \in L$, we define a complete congruence relation $\Theta_{a}$ of $K$.

If $a=0$, then $\Theta_{a}=\omega$, the trivial congruence. If $a=1$, then $\Theta_{a}=\iota$, the full 
congruence. Let $a \neq 0$ and $a \neq 1$. We define $\Theta_{a}^{C}$ as the smallest complete congruence relation of $C$ that collapses all prime intervals of $C$ of color $\leq a$. Similarly, we define $\Theta_{a}^{D}$ and $\Theta_{a}^{E}$. Then $\Theta_{a}^{C} \times \Theta_{a}^{D} \times \Theta_{a}^{E}$ is a complete congruence $\bar{\Theta}_{a}$ on the grid $C \times D \times E$. We can regard $\bar{\Theta}_{a}$ as a relation on $\hat{K}$; by making the classes convex, we get a complete congruence relation $\hat{\Theta}_{a}$ on $\hat{K}$.

$\hat{\Theta}_{a}$ is easy to visualize: if $p=\langle c, d, e\rangle$ is a grid element with a monochromatic prime square with respect to the axis $E$ over $p$, as in Figure 6 , and $c \varphi_{C}=d \varphi_{D} \leq a$, then the grid elements $\langle c, d, e\rangle,\left\langle c^{*}, d, e\right\rangle,\left\langle c, d^{*}, e\right\rangle,\left\langle c^{*}, d^{*}, e\right\rangle$ are in one $\bar{\Theta}_{a}$ class; so, under $\hat{\Theta}_{a}$, this class also contains $m(p, E)$. Moreover, if there is a monochromatic prime cube over $p$, and $c \varphi_{C}=d \varphi_{D} d=e \varphi_{E} \leq a$, then the eigth elements of the cube are in one class under $\bar{\Theta}_{a}$; so under $\hat{\Theta}_{a}$ this class also contains the elements: $m(p, C), m(p, D), m(p, E), m\left(p_{C}, C\right), m\left(p_{D}, D\right), m\left(p_{E}, E\right), a(p)$ and $b(p)$. This completely desribes the relation $\hat{\Theta}_{a}$.

We define $\Theta_{a}$ of $K$ as the restriction of $\hat{\Theta}_{a}$ from $\hat{K}$ to $K$.

Lemma 5. $\Theta_{a}$ is a complete congruence relation of $K$.

This is clear if $a=0$ or 1 , so we assume that $a \neq 0,1$. It is obvious that $\Theta_{a}$ is a congruence relation of $K$. By Lemma 2, in order to prove that it is a complete congruence relation, it is sufficient to prove all congruence classes of $\Theta_{a}$ are intervals.

To see this, take a congruence class $U$ of $\Theta_{a}$. Then $U$ is the restriction to $K$ of the convex sublattice of $\hat{K}$ generated by $U_{C} \times U_{D} \times U_{E}$, where $U_{C}$ is a congruence class of $\Theta_{a}^{C}$ of $C$, and similarly for $U_{D}$ and $U_{E}$. Since $a \neq 1$ and $1^{\delta} \varphi_{C}=1$, it follows that $U_{C}$ is contained in one $\left(X^{\delta}\right)^{\dagger}$.

There are two cases to consider. If $\bigvee X^{\delta} \not \leq a$, then, by our construction of $K$, $U_{D} \subseteq D^{\delta}$ - see Figures 9 and 8. Since each prime interval of $D^{\delta}$ is labeled with $\bigvee X^{\delta}$, this means $U_{D}=\{d\}$ for some $d \in D^{\delta}$. Therefore, $U=U_{C} \times\{d\} \times U_{E}$.

If $\bigvee X^{\delta} \leq a$, then $0^{\delta} \equiv j^{\delta}\left(\Theta_{a}\right)$. Since

$$
0^{\delta} \varphi_{C}=0^{D^{\delta}} \varphi_{D},
$$

it follows that we added the elements $m(p, E)$, for $p=\left\langle 0^{\delta}, d, e\right\rangle$, for all $d \in D^{\delta}$, $d<1^{D^{\delta}}$ and $e \in E$. Thus, for a fixed $e \in E$, all the elements $\left\langle 0^{\delta}, d, e\right\rangle$, for all $d \in D^{\delta}$ with $d<1^{D^{\delta}}$, are in one congruence class under $\Theta_{a}$. Since $\Theta_{a}$ is a complete congruence relation, and

$$
\bigvee\left(\left\langle 0^{\delta}, d, e\right\rangle \mid d \in D^{\delta} \text { and } d<1^{D^{\delta}}\right)=\left\langle 1^{\delta}, 1^{D^{\delta}}, e\right\rangle,
$$

it follows that

$$
U=\left[\left\langle 0^{\delta}, 0^{D^{\delta}}\right\rangle,\left\langle 1^{\delta}, 1^{D^{\delta}}\right\rangle\right] \times U_{E},
$$

completing the proof.

Notice that, since $\mathfrak{M}_{3}$ and the subspace lattice of the Fano plane are both simple lattices, all congruences classes of $\Theta_{a}$ are bounded by grid elements.

8. Proof of Theorem. We show that the correspondence

$$
\psi: x \rightarrow \Theta_{x}
$$

is an isomorphism between $L$ and $\operatorname{Con}^{c} K$, proving the Theorem. 
Lemma 6. $\psi$ is one-to-one and isotone.

It is trivial that $\psi$ is isotone. Let $b \not \leq a$ in $L$. Take an $e \in E$ with $e \varphi_{E}=b$. Then $e \equiv e^{*}\left(\Theta_{a}^{E}\right)$ fails, so $\Theta_{b} \not \Theta_{a}$.

Lemma 7. $\psi$ is onto.

We first show that the complete congruence generated by a prime interval of $K$ is of the form $\Theta_{a}$.

Obviously, every prime interval is projective to one in which two coordinates agree; such a prime interval is of the form $\mathfrak{p} \times\{d\} \times\{e\}$, where $\mathfrak{p}$ is a prime interval of $C, d \in D, e \in E$, or similarly, for a prime interval of $D$ or for a prime interval of $E$. Moreover, if two prime intervals of $K$ have the same color, they are projective in $K$. To see this, consider first the prime interval $\mathfrak{p} \times\{d\} \times\{e\}$, where $\mathfrak{p}=\left[p, p^{*}\right]$. Let $a$ be the color of $\mathfrak{p}$. By construction, there is a unique prime interval $\mathfrak{q}$ of $E$ with $\mathfrak{q} \varphi_{E}=a$ and if $\mathfrak{q}=\left[q, q^{*}\right]$, then $\mathfrak{p} \times\{d\} \times\{e\}$ and $\{p\} \times\{d\} \times \mathfrak{q}$ are projective using the element $m(\langle p, d, q\rangle, D)$. Now $\{p\} \times\{d\} \times \mathfrak{q}$ transposes down to $\left\{0^{C}\right\} \times\left\{0^{D}\right\} \times \mathfrak{q}$. Similarly, any prime interval of $D$ of color $a$ is projective to $\left\{0^{C}\right\} \times\left\{0^{D}\right\} \times \mathfrak{q}$. From this it easily follows that any two prime quotients of the same color are projective.

Let $\Theta$ be the complete congruence on $K$ generated by a prime interval. By the above, we may assume that this interval lies in $C \times D \times E$. Let $a$ be its color. Since all prime intervals of color $a$ are projective, they are all collapsed by $\Theta$. Suppose that $b \leq a$. We need to show that $\Theta$ collapses every prime interval of color $b$. Let $X^{\delta}=\{a, b\}$. Then the sublattice of $\mathfrak{M}_{\delta}$ with $E$ coordinate 0 , is diagrammed in Figure 2. The labeling given there is correct except that $c=a \vee b=a$. Since $\Theta$ collapses all intervals of color $a$, it collapses the one labeled with $c$ and thus all of the $\mathfrak{M}_{3}$ 's are collapsed. Since $\Theta$ is a complete congruence, this implies that the least and greatest elements of Figure 2 are collapsed. In particular, the intervals labeled $b$ are collapsed. Since all prime intervals with color $b$ are projective to one another, all of them are collapsed by $\Theta$.

From these arguments it follows that $\Theta_{a} \leq \Theta$. The reverse inclusion follows from the fact that $\Theta_{a}$ is a complete congruence (Lemma 5) containing the generating interval of $\Theta$.

For the final step of the proof, we prove the formula

$$
\bigvee\left(\Theta_{a} \mid a \in A\right)=\Theta_{\bigvee A}
$$

where $A \subseteq L-\{0\}, A \neq \varnothing$. Indeed,

$$
\bigvee\left(\Theta_{a} \mid a \in A\right) \leq \Theta_{\bigvee A}
$$

is obvious, because $\psi$ is isotone. To prove the reverse inclusion, choose $X^{\delta}=A$. By the preceding discussion, we can regard $\Theta_{\bigvee} A$ as the complete congruence generated by the prime interval $\mathfrak{p}_{\bigvee A}=\left[0^{\delta}, j^{\delta}\right] \times\left\{0^{D^{\delta}}\right\} \times\left\{0^{E}\right\}$, and each $\Theta_{a}, a \in A$, as the complete congruence generated by a prime interval $\mathfrak{p}_{a}=\mathfrak{q}_{a} \times\left\{0^{D^{\delta}}\right\} \times\left\{0^{E}\right\}$, where $\mathfrak{q}_{a}$ is any prime interval of $X^{\delta}$ of color $a$. It follows that $\bigvee\left(\Theta_{a} \mid a \in A\right)$ collapses 
$\left\langle j^{\delta}, 0^{D^{\delta}}, 0^{E}\right\rangle$ with $\left\langle u, 0^{D^{\delta}}, 0^{E}\right\rangle$, for all $u \in X^{\delta}$ with $u \geq j^{\delta}$. By the completeness of the congruence relation $\bigvee\left(\Theta_{a} \mid a \in A\right)$, it follows that

$$
\left\langle j^{\delta}, 0^{D^{\delta}}, 0^{E}\right\rangle \equiv\left\langle 1^{\delta}, 1^{D^{\delta}}, 0^{E}\right\rangle \quad\left(\bigvee\left(\Theta_{a} \mid a \in A\right)\right)
$$

Meeting both sides with $\left\langle 0^{\delta}, v, 0^{E}\right\rangle$, where $v$ is the atom of $D^{\delta}$, we obtain that

$$
\left\langle 0^{\delta}, 0^{D^{\delta}}, 0^{E}\right\rangle \equiv\left\langle 0^{\delta}, v, 0^{E}\right\rangle \quad\left(\bigvee\left(\Theta_{a} \mid a \in A\right)\right)
$$

Since the prime interval $\left\{0^{\delta}\right\} \times\left[0^{D^{\delta}}, v\right] \times\left\{0^{E}\right\}$ of $K$ is of color $\bigvee A$, it is projective to $\mathfrak{p} \vee A_{A}$; hence $\Theta \bigvee A \leq \bigvee\left(\Theta_{a} \mid a \in A\right)$. This completes the proof of the formula.

Since by Lemma 1 every complete congruence of $K$ is a join of complete congruences generated by prime intervals, this formula shows that $\psi$ is onto.

This completes the proof of Lemma 7, and the proof of the Theorem.

9. Concluding Remarks. The lattice $K$ constructed to prove Theorem 1 has some rather special properties.

$K$ satisfies Huhn's 3-distributivity law, defined in [5]. It also satisfies Jónsson's arguesian law, defined in [6].

Recall that in any modular lattice $M$, if $\mathfrak{p}$ is a prime interval, $\Theta(\mathfrak{p})$ is an atom of the congruence lattice of $M$. In the proof, we found that every congruence on $K$ can be represented in the form $\Theta^{c}(\mathfrak{p})$, for some prime interval $\mathfrak{p}$. This shows how different congruences and complete congruences are for modular lattices.

Note that if $K$ is a complete distributive lattice, then $\Theta(\mathfrak{p})=\Theta^{c}(\mathfrak{p})$. This follows from Lemma 2, since every block of $\Theta(\mathfrak{p})$ has only one or two elements. Consequently, it is not possible to represent any complete lattice with no atom as the lattice of complete congruence relations of a complete distributive lattice with a prime interval.

\section{REFERENCES}

1. P. Crawley and R. P. Dilworth, Algebraic Theory of Lattices, Prentice-Hall, Englewood Cliffs, New Jersey, 1973.

2. G. Grätzer, General Lattice Theory, Academic Press, New York, N. Y., 1978; Birkhäuser Verlag, Basel; Akademie Verlag, Berlin.

3. G. Grätzer, The complete congruence lattice of a complete lattice., Proceedings of the International Conference on Universal Algebra, Lattices, and Semigroups (Lisbon, 1988).

4. G. Grätzer and H. Lakser, On complete congruence lattices of complete lattices, Trans. Amer. Math. Soc. (to appear).

5. A. P. Huhn, Schwach distributive Verbände, I, Acta Sci. Math. (Szeged) 33 (1972), 297-305.

6. B. Jónsson, Modular lattices and Desargues theorem, Math. Scand. 2 (1954), 295-314.

7. R. McKenzie, Equational bases and non-modular lattice varieties, Trans. Amer. Math. Soc. 174 (1972), 1-43.

8. S.-K. Teo, Representing finite lattices as complete congruence lattices of complete lattices, Abstracts of papers presented to the Amer. Math. Soc. 88T-06-207; Ann. Univ. Sci. Budapest. Eötvös Sect. Math. (to appear).

Department of Mathematics, University of Hawail Honolulu, Hawail 
E-mail address: ralph@kahuna.math.hawaii.edu

Department of Mathematics, University of Manitoba

Winnipeg, Man. R3T 2N2, CANADA

E-mail address: gratzer@ccm.umanitoba.ca, gratzer@uofmcc.bitnet

Mathematical Resereach Institure of the Hungarian Academy of Sciences Budapest, Hungary

E-mail address: h1175sch\%ella@relay.eu.net 